\title{
Psychological Reactions of Victims of Violent Crime
}

\author{
JONATHAN I. BISSON and JONATHAN P. SHEPHERD
}

Violent crime is on the increase. The 1992 British Crime Survey (Mayhew et al, 1993) revealed that $4.9 \%$ of men and $1.9 \%$ of women experienced violent crime (excluding domestic and sexual assaults) in 1991 . This represented a $24 \%$ increase on the 1981 figures. The true incidence is likely to be much higher. Hough \& Mayhew (1985) found that only $23 \%$ of woundings, $11 \%$ of robberies and $26 \%$ of sexual offences were recorded in police crime statistics. Research in accident and emergency departments (A\&Es) has confirmed the magnitude of the 'dark figure' of unrecorded violent crime (Shepherd et al, 1987).

The Epidemiologic Catchment Area survey (Helzer et al, 1987) found that $2.8 \%$ of a random sample of 2493 US adults were physically assaulted during the 18-month study period. Breslau et al (1991) studied 1007 young American adults of both sexes: $8.3 \%$ reported having been physically assaulted, and $1.6 \%$ having been raped.

Some individuals are at increased risk of becoming victims of violence through their work. These include the police, bank employees and health professionals, particularly family practitioners, A\&E doctors and nurses, and ambulance staff. O'Sullivan \& Meagher (1995) surveyed 178 psychiatrists and found that 39\% had been assaulted at work and $12 \%$ had been physically injured as a result.

The psychological reactions of victims of violent crime have much in common with those experienced by victims of other traumatic events, including major disasters. Although research on other traumatic stressors should stimulate the development of hypotheses that can be tested in studies of victims of violent crime, Green (1982) has cautioned against overgeneralisation between different traumas in the field of traumatic stress.

\section{Psychological sequelae of violent crime}

Any traumatic event, including violent crime, may precipitate an acute psychological response. Characteristic features of this include fear, anger, recurrent distressing thoughts, guilt, depression, anxiety, bad dreams, irritability and generalised hyperarousal. Such a response should be considered normal and has been described as such by various authors in the immediate aftermath of a violent crime.
Symonds (1975) described a classic four-stage reaction specific to victims of violence. Initial shock and denial are followed by fright and fear. Apathy and anger ensue, often with feelings of guilt and depression, followed by resolution or repression. The similarities between this, Horowitz's (1974) information processing model and the normal grief reaction are apparent. Symonds' stages may be telescoped together and be more difficult to identify in people subjected to repeat victimisation (Shepherd, 1990).

The response to violent crime can become problematic at any stage. A severe initial response often represents an acute stress disorder. With time, other conditions such as post-traumatic stress disorder (PTSD), anxiety disorders, depressive disorders and substance abuse/dependence may develop. These conditions can have devastating effects on victims' lives and markedly affect their functioning at personal, social and occupation levels.

\section{Prevalence of psychological sequelae}

Most research in the area of violent crime has focused on the psychological effects of sexual assaults on females. Rothbaum et al (1992) prospectively studied 95 female rape victims and found that $47 \%$ of them met the criteria for PTSD three months after the rape. Lopez et al (1992) described a retrospective questionnaire survey of $\mathbf{4 3 6}$ rape victims in which $71 \%$ reported depression and $37.5 \%$ chronic PTSD. Breslau et al (1991) found that $22.6 \%$ of those physically assaulted and $80 \%$ of rape victims developed PTSD. Helzer et al (1987) found the prevalence of PTSD to be much lower. In their study only $2.9 \%$ of those who had been physically assaulted in the preceding 18 months met the criteria for a diagnosis of PTSD.

Shepherd et al (1990) studied 122 consecutive patients who were treated for jaw fractures in Bristol. Seventy of the fractures were the result of an assault, and the remainder were sustained in accidents. Between $10 \%$ and $15 \%$ of both groups scored greater than 10 on the anxiety and depression scales of the Hospital Anxiety and Depression Scale (HADS) one week after the trauma. Although the accident group showed a significant reduction in their HADS scores by three months, levels of anxiety and depression had not decreased in the assault group. 


\section{Factors associated with psychological sequelae}

Breslau et al (1991) found that the prevalence of psychological sequelae following physical assault were similar for men and women. However, women were more affected than men in the groups who had witnessed someone else being killed or seriously hurt. Because the prevalence of PTSD in rape victims was found to be $80 \%$, these workers concluded that, overall, PTSD prevalence after a major traumatic event was $100 \%$ higher in women than in men.

In keeping with the findings of research on major traumatic events other than violent crime, the greater the severity of the stressor the more likely psychological sequelae are to ensue. Resnick et al (1992) compared 'high crime stress' with 'low crime stress' in a community sample of 295 female crime victims. They found a much higher rate of PTSD among the 'high crime stress' group (35\% v. 13\%). The dimensions they found to be particularly associated with a greater risk of PTSD were threat to life or physical integrity, physical injury, receipt of intentional harm, exposure to grotesque sights, violent or sudden death of a loved one, subjective perception of threat, and completed rape. These factors were far more important in determining psychological sequelae than 'pre-crime' factors. For example, pre-crime depression was associated with the development of PTSD only in the group experiencing high crime stress. These findings are not consistent with the hypothesis that the more vulnerable the individual the less stress it takes to precipitate PTSD.

Kilpatrick et al (1989) also found that the circumstances of crime were more important predictors for PTSD. They performed a hierarchical multiple regression analysis on 294 female crime victims and discovered that life threat, physical injury and completed rape were the most important predictors. Indeed, with all three elements present in an assault there was an 8.5-fold increased risk of PTSD than if none was present.

Across a wide spectrum of traumatic events, however, there is good evidence that variables other than the dimensions of the trauma itself do influence outcome. An acute stress disorder, a psychiatric history, a family psychiatric history, lack of social support and high 'neuroticism' have all been associated with an increased rate of PTSD. Further research is required to establish the importance of these factors in responses to violent crime.

\section{Management}

Studies have suggested that victims of crime are difficult to engage in treatment (Koss \& Burkhart, 1989).
The attitude of the victim towards help is critically important and is likely to depend on social and cultural influences as well as personality. Consideration of the varied individual responses that arise from deeply held beliefs about what is the correct or expected behaviour should help in the provision of effective and acceptable interventions.

Various interventions have been used with the victims of violent crime and can be usefully divided into preventive and treatment strategies.

\section{Prevention}

Prevention following a violent crime has the objective of reducing the incidence of psychological sequelae through the use of early interventions. Central to this is the assumption that talking through what happened helps victims to process traumatic events. There remains a paucity of empirical evidence confirming the benefits of very brief early interventions such as psychological debriefing (Bisson \& Deahl, 1994), but many victims value the opportunity to talk about their emotions and may also benefit from practical advice.

In Britain, the national charity Victim Support provides emotional support and offers practical help to victims of crime. It contacted 175733 victims of violent crime in the 12 months to March 1994 (Victim Support, 1994).

Employers also have an important role to play in prevention. Routine training in the basics of trauma psychology and provision for employees' needs following crime should help to reduce psychological sequelae. An adequate complaints procedure, the opportunity to talk through what has happened, help with compensation claims and time off are all likely to help victims process their trauma.

\section{Treatment}

For a sizeable minority of victims of violent crime, reactions can be considered pathological and treatment is needed. It is crucially important that psychiatric disorder is detected and treated as soon after the event as possible. McFarlane (1984) criticised overzealous normalisation attempts after the Ash Wednesday Australian bush fires in 1983. The same applies to victims of crime. There is a real danger that over-normalisation can result in psychiatric disorders being missed and appropriate treatment being withheld because symptoms are mistakenly considered 'understandable'.

Distinct clinical disorders should be treated in their own right - for example depression with antidepressants or cognitive-behavioural therapy. It is 
also important to consider the violent crime itself. Exposure therapy and medication have been shown to help PTSD sufferers in randomised clinical trials (Solomon et al, 1992). Exposure therapy involves reconsidering the traumatic event in great detail, often with imaginal exposure to the event itself and real-life exposure to fear-provoking cues. Various antidepressants have been shown to be useful, including phenelzine, amitriptyline and fluoxetine. Other therapies are also likely to help but have not yet been adequately assessed. Cognitive therapy, psychodynamic therapy, group therapy and hypnosis have all been reported to have helped PTSD sufferers.

There are few studies that consider the treatment of victims of crime specifically. Perhaps the best known was performed by Foa et al (1991), who compared exposure therapy with stress inoculation training, supportive counselling and a waiting-list control group. They found that stress inoculation training produced the best results immediately after therapy and that those who received exposure therapy fared best at three-month follow-up. Not surprisingly, a study of the effectiveness of a combination of these approaches has resulted.

\section{Conclusion}

There is an urgent need for rigorous evaluation of the effectiveness of interventions and ways of recruiting victims of violent crime into helpful initiatives. The development of appropriate services should then ensue. Voluntary organisations, such as Victim Support, and health professionals in primary and secondary care are all likely to play key roles. A\&E departments should be the focus for initiatives in the hospital setting, because the majority of victims who attend for treatment are not known to the police and are therefore unlikely to be referred to Victim Support.

It is appropriate to offer all victims of violent crime early emotional support, education and practical advice. If symptoms do not improve within four to six weeks or deteriorate, more active treatment should be offered.

\section{References}

Bisson, J. I. \& Deahl, M. (1994) Psychological debriefing and prevention of post-traumatic stress. British Journal of Psychiatry, 165, 717-720.
Breslau, N., Davis, G. C., Andreski, P., et al (1991) Traumatic events and posttraumatic stress disorder in an urban population of young adults. Archives of General Psychiatry, 48, 216-222.

Fon, E. B., RothiuUm, B. O., Ricos, D. S., et al (1991) Treatment of posttraumatic stress disorder in rape victims: a comparison between cognitive-behavioural procedures and counselling. Journal of Consulting and Clinical Psychology, 59, 715-723.

GreEN, B. L. (1982) Assessing levels of psychological impairment following disaster: consideration of actual and methodological dimensions. Journal of Nervous and Mental Disease, 170, 544-552.

Helzer, J. E., Robins, L. N. \& McEvor, L. (1987) Post-traumatic stress disorder in the general population: findings of the Epidemiologic Catchment Area survey. New England Journal of Medicine, 317, 1630-1634.

HorowrTz, M. (1974) Stress response syndromes: character style and dynamic psychotherapy. Anchives of General Psychiatry, 31, 768-781.

Hough, M. \& MAYHEw, P. (1985) Taking Account of Crime: Key Findings from the 1984 British Crime Survey. (Home Office research study no. 85.) London: HMSO.

Kilpatrick, A. G., Saunders, B. E., Amick-McMullan, A., et al (1989) Victim and crime factors associated with the development of crime related post traumatic stress disorder. Behaviour Therapy, 20, 199-214.

Koss, M. P. \& Burkhart, B. R. (1989) A conceptual analysis of rape victimisation. Psychology of Women Quarterly, 13, 27-40.

Lopez, G., Piffaut, G. \& Seguin, A. (1992) Psychological treatment of victims of rape. Psychological Medicine, 24, 286-288.

Mayhew, P., Maung, N. A. \& Mirrlees-Black, C. (1993) The 1992 British Crime Survey. London: HMSO.

MCFARLANE, A. (1984) The Ash Wednesday bushfires in South Australia: implications for planning for future post-disaster services. Medical Journal of Australia, 141, 286-291.

O'Sullivan, M. \& MeAGHer, D. (1995) A 3 year prospective audit of assaults against psychiatrists and trainees in Dublin. Abstracts of the 1995 Winter Meeting of the Royal College of Psychiatrists. London: Royal College of Psychiatrists.

Resnick, H. S., Killpatrick, D. G., Best, C. L., et al (1992) Vulnerability-stress factors in development of post traumatic stress disorder. Journal of Nervous and Mental Disease, 180, 424-430.

Rothbaum, B. O., Fon, E. B., Murdock, T., et al (1992) A prospective examination of post-traumatic stress disorder in rape victims. Journal of Traumatic Stress, 5, 455-475.

ShePherd, J. P. (1990) Personal violence: the relevance of Symond's model of psychological response and loss theory. British Journal of Social Work, 20, 309-332.

-, Pierce, N. X., Scully, C., et al (1987) Rates of violent crime from hospital records. Lancet, $i i, 1470-1471$.

- , QUReshi, R., Preston, M. S., et al (1990) Psychological distress after assaults and accidents. British Medical Journal, 301, 849-850.

Solomon, S. D., Gerrity, E. T. \& Muff, A. M. (1992) Efficacy of treatments for posttraumatic stress disorder. Journal of the American Medical Association, 268, 633-638.

SyMONDS, M. (1975) Victims of violence: psychological effects and after effects. American Journal of Psychoanalysis, 35, 19-26. Victim SUPPORT (1994) The 1994 Annual Report. London: Victim Support.

Jonathan I. Bisson, MRCPsych, Jonathan P. Shepherd, MSc, University of Wales College of Medicine, Cardiff

Correspondence: Dr Jonathan I. Bisson, Department of Psychological Medicine, University of Wales College of Medicine, Heath Park, Cardiff CF4 4XN

(First received 23 March 1995, final revision 22 May 1995, accepted 8 June 1995) 